\title{
INTEGER POINTS ON ALGEBRAIC CURVES WITH EXCEPTIONAL UNITS
}

\author{
DIMITRIOS POULAKIS
}

(Received 8 January 1996; revised 12 June 1996)

\author{
Communicated by W. W. L. Chen
}

\begin{abstract}
Let $F(X, Y)$ be an absolutely irreducible polynomial with coefficients in an algebraic number field $K$. Denote by $C$ the algebraic curve defined by the equation $F(X, Y)=0$ and by $K[C]$ the ring of regular functions on $C$ over $K$. Assume that there is a unit $\varphi$ in $K[C]-K$ such that $1-\varphi$ is also a unit. Then we establish an explicit upper bound for the size of integral solutions of the equation $F(X, Y)=0$, defined over $K$. Using this result we establish improved explicit upper bounds on the size of integral solutions to the equations defining non-singular affine curves of genus zero, with at least three points at 'infinity', the elliptic equations and a class of equations containing the Thue curves.
\end{abstract}

1991 Mathematics subject classification (Amer. Math. Soc.): primary 11D41, $11 \mathrm{G} 30$.

\section{Introduction}

Let $K$ be an algebraic number field, $O_{K}$ its ring of integers and $\bar{K}$ an algebraic closure of $\mathrm{K}$. Let $F(X, Y)$ be an absolutely irreducible polynomial in $K[X, Y]$. Denote by $C$ the algebraic curve defined by the equation $F(X, Y)=0$ and by $\bar{K}[C]$ the ring of regular functions on $C$. We shall call a unit $\varphi$ in $\bar{K}[C]-\bar{K}$ exceptional, if $1-\varphi$ is also a unit. The ring of regular functions of a wide class of algebraic curves contains exceptional units. For instance the Thue curves ([7, p. 144]) and the modular curves $X(N)$ ([6, ch. 8], [5]) have this property. In this paper we assume that $K[C]$ contains an exceptional unit and we calculate an explicit upper bound on the size of solutions $(x, y) \in O_{K}^{2}$ to the equation $F(X, Y)=0$. This result enables us to establish improved explicit upper bounds on the size of integral solutions to the equations defining non-singular affine curves of genus 0 , with at least three points at 'infinity', the elliptic equations and a class of equations containing the Thue equations.

(c) 1997 Australian Mathematical Society 0263-6115/97 \$A2.00+0.00 
In Section 2 a particular effective version of Hilbert's Nullstellensatz and an upper bound for the solutions of the linear equation in algebraic integers of bounded norm, permit us to establish an explicit upper bound on the size of integer points on curves which the ring of regular functions has an exceptional unit $\varphi$. This bound depends on the polynomial $F(X, Y)$, the field $K$ and the function $\varphi$. In the next sections we give some applications. In Section 3 we prove that the ring of regular functions of a non-singular affine curve of genus 0 , with at least three points at 'infinity', has an exceptional unit and we deduce an explicit upper bound on the size of their integer points, improving on the bound given in [14]. In Section 4, using 'multiplication by 2' on an elliptic curve, we reduce the problem of calculating an explicit upper bound on the size of integral solutions of an elliptic equation to the same problem for a curve having an exceptional unit. Thus we deduce an explicit upper bound on the size of integral solutions of the elliptic equation, improving on Schmidt's estimate [17]. In Section 5 we consider the class of irreducible curves of the form

$$
\left(X-a_{1} Y\right)\left(X-a_{2} Y\right)\left(X-a_{3} Y\right) f(X, Y)+b=0,
$$

where $f(X, Y) \in K[X, Y], a_{1}, a_{2}, a_{3}, b \in K$ and the elements $a_{1}, a_{2}, a_{3}$ are pairwise distinct. We prove that the curves of this class have exceptional units. Thus we deduce an upper bound on the size of their integer points. We note that the Thue curves belong to this class.

Let $\tilde{C}$ be a non-singular model of $C$. We denote by $\Sigma$ the set of poles of the function defined by $X$ on $\tilde{C}$. If $\bar{K}[C]$ has an exceptional unit $\varphi$, then we easily deduce that the rank of the group of $\Sigma$-units on $\tilde{C}$ is $\geq 2$. Therefore, [1, Theorem 1B] gives an effective bound for the size of $S$-integer solutions of the equation $F(X, Y)=0$, over $K$ (see also [2]). This bound is not in completely explicit form and does not depend on the unit $\varphi$. Furthermore, in [1], effective bounds are given, not in completely explicit form, for the size of $S$-integer points on the affine models of curves of genus 0 , with at least three points at 'infinity' and Thue curves. In the case of integer points, our estimates are sharper than those of [1].

NotaTION. Throughout this paper we denote by $d, D_{K}$ and $N_{K}$ respectively the degree of $K$, the discriminant of $K$ and the norm from $K$ to the field $\mathbb{Q}$ of rationals. By an absolute value we will always understand an absolute value that it extends either the standard absolute value of $\mathbb{Q}$ or a p-adic absolute value $|\cdot|_{p}$ of $\mathbb{Q}$. Let $M(K)$ be a set of symbols $v$ such that with every $v \in M(K)$ there is associated precisely one absolute value $|\cdot|_{v}$. We denote by $d_{v}$ the local degree of $|\cdot|_{v}$. Let $\mathbf{x}=\left(x_{0}: \ldots: x_{n}\right)$ be a point in a projective $n$-space $P^{n}(K)$ over $K$. We define the field height of $\mathbf{x}$ to be

$$
H_{K}(\mathbf{x})=\prod_{v \in M(K)} \max \left\{\left|x_{0}\right|_{v}, \ldots,\left|x_{n}\right|_{v}\right\}^{d_{v}}
$$


and its absolute height to be $H(\mathbf{x})=H_{K}(\mathbf{x})^{1 / d}$. For $x \in K$ we define $H_{K}(x)=$ $H_{K}((1: x))$ and $H(x)=H((1: x))$. Let $G$ be a polynomial in one or several variables and with coefficients in $K$. We define the field height $H_{K}(G)$ and the absolute height $H(G)$ of $G$ to be respectively the field height and the absolute height of a point in a projective space having as coordinates the coefficients of $G$ (in any order). Given $v \in M(K)$, we denote by $|G|_{v}$ the maximum $|c|_{v}$ over all the coefficients $c$ of $G$. For an account of the properties of heights see [18, ch. VIII] and [9, ch. 3]. Finally, for a positive real number $z$, we let $\log * z=\max \{1, \log z\}$.

\section{Curves with exceptional units}

In this section we calculate an explicit upper bound for the size of integer points on curves having exceptional units. Let $F(X, Y)$ be an absolutely irreducible polynomial in $K[X, Y]$ of degree $N \geq 2$ and $C$ the algebraic curve defined by the equation $F(X, Y)=0$. Denote by $K[C]$ the ring of regular functions of $C$ defined over $K$. We shall prove the following result:

THEOREM 1. Let $F(X, Y)$ and $C$ be as above. Suppose that there exists an exceptional unit $\varphi$ in $K[C]-K$. Let $f(X, Y)$ be a polynomial in $K[X, Y]$ representing the function $\varphi$ on $C$. Put $\Delta=\max \{\operatorname{deg} f, N\}$ and let $\Lambda$ be a point in a projective space having as coordinates 1 and the coefficients of the polynomials $f(X, Y)$ and $F(X, Y)$ (in any order). Then all solutions $x, y \in O_{K}$ of the equation $F(X, Y)=0$ satisfy

$$
\max \left\{H_{K}(x), H_{K}(y)\right\}<\exp \left\{\Omega_{1}(d, \Delta)\left|D_{K}\right|\left(\log *\left|D_{K}\right|\right)^{2 d} \log * H_{K}(\Lambda)\right\}
$$

where $\Omega_{1}(d, \Delta)<\Delta^{6} d^{11 d+13} 81^{d+9}$.

The following results will be useful for the proof of Theorem 1 .

LEMMA 1. Let $a_{1}, \ldots, a_{n} \in K$. Then there is $\beta \in O_{K}$ such that $\beta a_{1}, \ldots, \beta a_{n} \in O_{K}$ and

$$
H_{K}(\beta) \leq\left|D_{K}\right|^{1 / 2} H_{K}(A)
$$

where $A$ is a point in a projective space having as coordinates 1 and $a_{1}, \ldots, a_{n}$.

ProOF. Consider the polynomial $f(X)=a_{n} X^{n}+\cdots+a_{1} X+1$ and a positive integer $m \geq 2$. By [19, Lemma 2.(i)], we deduce that there is $\beta \in O_{K}$ such that $\beta f(X) \in O_{K}[X]$ and

$$
H_{K}\left(Y^{m}-\beta f(X)\right) \leq\left|D_{K}\right|^{1 / 2} H_{K}\left(Y^{m}-f(X)\right)
$$


Then

$$
H_{K}(\beta) \leq H_{K}\left(Y^{m}-\beta f(X)\right) \leq\left|D_{K}\right|^{1 / 2} H_{K}(A) .
$$

LEMMA 2. Let the polynomials $P, R \in O_{K}[X, Y]-K$ have no common zero in $\bar{K}^{2}$. Assume that the degrees of $P$ and $R$ are at most $D \geq 2$. Denote by $\Phi$ a point in a projective space having as coordinates the coefficients of $P$ and $R$. Then there are polynomials $A, B \in O_{K}[X, Y]$ and $c \in O_{K}, c \neq 0$, satisfying

$$
A P+B R=c,
$$

such that

$$
\operatorname{deg} A, \operatorname{deg} B \leq 4 D^{2}+2 D
$$

and for every archimedean absolute value $|\cdot|_{v}$ of $K$

$$
|A|_{v},|B|_{v},|c|_{v} \leq\left(49 D^{4}\right) !|\Phi|_{v}^{49 D^{4}} .
$$

Proof. By [3, Theorem 1], there are polynomials $A, B \in K[X, Y]$ satisfying $A P+B R=1$, such that $\operatorname{deg} A, \operatorname{deg} B \leq 4 D^{2}+2 D$. Write

$$
\begin{aligned}
& A=\sum_{i=0 . j=0}^{a, a^{\prime}} A_{i j} X^{i} Y^{j}, \quad B=\sum_{i=0, j=0}^{b, b^{\prime}} B_{i j} X^{i} Y^{j}, \\
& P=\sum_{i=0, j=0}^{p, p^{\prime}} P_{i j} X^{i} Y^{j}, \quad R=\sum_{i=0, j=0}^{r, r^{\prime}} R_{i j} X^{i} Y^{j}
\end{aligned}
$$

Then

$$
\sum_{s=0, t=0}^{S, T}\left(\sum_{i+k=s, j+l=t}\left(A_{i j} P_{k l}+B_{i j} R_{k l}\right)\right) X^{s} Y^{t}=1,
$$

where $S=\max \{a+p, b+r\}$ and $T=\max \left\{a^{\prime}+p^{\prime}, b^{\prime}+r^{\prime}\right\}$. It follows that the numbers $A_{i j}\left(i=0, \ldots, a, j=0, \ldots, a^{\prime}\right), B_{i j}\left(i=0, \ldots, b, j=0, \ldots, b^{\prime}\right)$ and 1 are a solution of the homogeneous linear system

$$
\begin{aligned}
\sum_{i+k=s, j+l=t} X_{i j} P_{k l}+Y_{i j} R_{k l} & =0 \quad(s=1, \ldots, S, t=1, \ldots, T) \\
X_{00} P_{00}+Y_{00} R_{00}-Z & =0,
\end{aligned}
$$

in unknowns $X_{i j}\left(i=0, \ldots, a, j=0, \ldots, a^{\prime}\right), Y_{i j}\left(i=0, \ldots, b, j=0, \ldots, b^{\prime}\right)$ and $Z$. There are at most $1+49 D^{4}$ equations and at most $75 D^{4}$ unknowns. By 
the proof of $\left[11\right.$, p. 442, Lemma 4], there is a non-trivial solution $\gamma_{i j}(i=0, \ldots, a$, $\left.j=0, \ldots, a^{\prime}\right), \epsilon_{i j}\left(i=0, \ldots, b, j=0, \ldots, b^{\prime}\right)$ and $c$ in $O_{K}$, such that $c \neq 0$ and for every archimedean absolute value $|\cdot|_{v}$ of $K$

$$
\left|\gamma_{i j}\right|_{v},\left|\epsilon_{i j}\right|_{v},|c|_{v} \leq\left(49 D^{4}\right) !|\Phi|_{v}^{49 D^{4}}
$$

where $\Phi$ is a point in a projective space having as coordinates the coefficients of $P$ and $R$. Moreover the polynomials

$$
\Gamma=\sum_{i=0, j=0}^{a . a^{\prime}} \gamma_{i j} X^{i} Y^{j} \quad \text { and } \quad E=\sum_{i=0 . j=0}^{b . b^{\prime}} \epsilon_{i j} X^{i} Y^{j}
$$

satisfy $\Gamma P+E R=c$.

LEMMA 3. Assume $d \geq 2$. Let $a, b$ be two non-zero elements of $K$ with absolute heights at most $A \geq e$. Let $M$ be a positive integer. Then all solutions $x, y \in O_{K}-\{0\}$ of the equation $a X+b Y=1$, such that $\max \left\{N_{K}(x), N_{K}(y)\right\} \leq M$, satisfy

$$
\max \left\{H_{K}(x), H_{K}(y)\right\}<\exp \left\{\Psi(d)\left|D_{K}\right|\left(\log *\left|D_{K}\right|\right)^{2 d-1} \log (M A)\right\},
$$

where

$$
\Psi(d)<11 d^{11 d+13} 81^{d+7} .
$$

PROOF. There exists a positive constant $\delta_{K}$, depending on $K$, such that for every $a \in K-\{0\}$ which is not a root of unity we have $\log H(a) \geq \delta_{K} / d$. It follows from [20] that we can take $\delta_{K}=2 /(\log 3 d)^{3}$. Let $R_{K}$ be the regulator of $K$. By [10],

$$
R_{K}<C_{1}(d)\left|D_{K}\right|^{1 / 2}\left(\log *\left|D_{K}\right|\right)^{d-1},
$$

where

$$
C_{1}(d)=\frac{\left(1+1 / \log \left|D_{K}\right|\right)^{d+1} d \sqrt{e}}{(2 \sqrt{\pi})^{d}} \leq \frac{5 d}{1.45^{d}}<5 .
$$

Let $x, y \in O_{K}-\{0\}$ with $\max \left\{N_{K}(x), N_{K}(y)\right\} \leq M$ satisfying $a x+b y=1$. By [4, Corollary] there is a unit $\epsilon$ in $O_{K}$ such that

$$
\max \{H(\epsilon x), H(\epsilon y), H(\epsilon)\}<\exp \left\{C_{2}(d) R_{K}\left(\log * R_{K}\right)\left(R_{K}+\log (M A)\right)\right\},
$$

where

$$
C_{2}(d)<\frac{1}{2} \delta_{K}^{1-2 d} 3^{2 d+28} d^{8 d+12}
$$


We easily deduce $\delta_{K}^{1-2 d} \leq 3^{2 d-1} d^{3 d-1}$ and

$$
R_{K}\left(\log * R_{K}\right)\left(R_{K}+\log (M A)\right)<33 d\left|D_{K}\right|\left(\log *\left|D_{K}\right|\right)^{2 d-1} \log (M A) .
$$

Then combining the above inequalities, we get

$$
\max \{H(\epsilon x), H(\epsilon y), H(\epsilon)\}<\exp \left\{C_{3}(d)\left|D_{K}\right|\left(\log *\left|D_{K}\right|\right)^{2 d-1} \log (M A)\right\},
$$

where $C_{3}(d)<\left(11 d^{11 d+12} 81^{d+7}\right) / 2$.

We have

$$
H(x)=H(x \epsilon / \epsilon) \leq H(x \epsilon) H\left(\epsilon^{-1}\right) \leq H(x \epsilon) H(\epsilon) .
$$

Similarly $H(y) \leq H(y \epsilon) H(\epsilon)$. Thus we deduce

$$
\max \left\{H_{K}(x), H_{K}(y)\right\}<\exp \left\{\Psi(d)\left|D_{K}\right|\left(\log *\left|D_{K}\right|\right)^{2 d-1} \log (M A)\right\},
$$

where $\Psi(d)<11 d^{11 d+13} 81^{d+7}$.

LEMMA 4. Let $F(X)$ be a polynomial in $K[X]-\{0\}$. If a is an algebraic number such that $F(a)=0$, then we have $H(a)<2 H(F)$.

PROOF. Write $F(X)=c_{0} X^{n}+c_{1} X^{n-1}+\cdots+c_{n}$. Then $F(a)=c_{0} a^{n}+c_{1} a^{n-1}+$ $\cdots+c_{n}=0$. Let $|\cdot|_{v}$ be an absolute value of $K(a)$. When $|\cdot|_{v}$ is non-archimedean, we have

$$
\max \left\{1,|a|_{v}\right\} \leq \max \left\{\left|c_{1} / c_{0}\right|_{v}, \ldots,\left|c_{n} / c_{0}\right|_{v}, 1\right\}
$$

and when $|\cdot|_{v}$ is archimedean, [13, Corollary 2] gives

$$
\max \left\{1,|a|_{v}\right\}<1+\max \left\{\left|c_{1} / c_{0}\right|_{v}, \ldots,\left|c_{n} / c_{0}\right|_{v}, 1\right\} .
$$

Thus we obtain

$$
H_{K(a)}(a)<2^{\operatorname{deg} K(a)} H_{K(a)}(F) .
$$

LEMMA 5. Let $f(X, Y), g(X, Y)$ be non-zero polynomials in $K[X, Y]-K$ and $R(X)$ the resultant of $f(X, Y), g(X, Y)$ considered as polynomials with coefficients in $K[X] . \quad$ Put $\operatorname{deg}_{Y} f=n_{1}, \operatorname{deg}_{X} f=m_{1}, \operatorname{deg}_{Y} g=n_{2}, \operatorname{deg}_{X} g=m_{2}$. Assume $R(X) \not \equiv 0$. Then

$$
H(R) \leq\left(n_{1}+n_{2}\right) !\left(m_{1}+1\right)^{n_{2}}\left(m_{2}+1\right)^{n_{1}} H(f)^{n_{2}} H(g)^{n_{1}} .
$$


PROOF. Write $f(X, Y)=f_{n_{1}}(X) Y^{n_{1}}+\cdots+f_{0}(X)$ and $g(X, Y)=g_{n_{2}}(X) Y^{n_{2}}+$ $\cdots+g_{0}(X)$, where $f_{i}(X), g_{j}(X) \in K[X]\left(i=0, \ldots, n_{1}, j=0, \ldots, n_{2}\right)$. The polynomial $R(X)$ is homogeneous of degree $n_{2}$ in $f_{n_{1}}(X), \ldots, f_{0}(X)$ and of degree $n_{1}$ in $g_{n_{2}}(X), \ldots, g_{0}(X)$ with rational integer coefficients. If $|\cdot|_{v}$ is a non-archimedean absolute value, then we get $|R|_{v} \leq|f|_{v}^{n_{2}}|g|_{v}^{n_{1}}$. Let $|\cdot|_{v}$ be an archimedean absolute value. If $M(X)$ is a monomial of degree $n_{2}$ in $f_{n_{1}}(X), \ldots, f_{0}(X)$ and of degree $n_{1}$ in $g_{n_{2}}(X), \ldots, g_{0}(X)$, then we deduce

$$
|M(X)|_{v} \leq\left(m_{1}+1\right)^{n_{2}}\left(m_{2}+1\right)^{n_{1}}|f|_{v}^{n_{2}}|g|_{v}^{n_{1}} .
$$

Thus we obtain

$$
|R|_{v} \leq\left(n_{1}+n_{2}\right) !\left(m_{1}+1\right)^{n_{2}}\left(m_{2}+1\right)^{n_{1}}|f|_{v}^{n_{2}}|g|_{v}^{n_{1}} .
$$

Therefore

$$
H(R) \leq\left(n_{1}+n_{2}\right) !\left(m_{1}+1\right)^{n_{2}}\left(m_{2}+1\right)^{n_{1}} H(f)^{n_{2}} H(g)^{n_{1}} .
$$

ProOF OF THEOREM 1. By Lemma 1, there is $\delta \in O_{K}$ such that the polynomials $f_{1}(X, Y)=\delta f(X, Y)$ and $F_{1}(X, Y)=\delta F(X, Y)$ have all their coefficients in $O_{K}$. Further, the height of $\delta$ satisfies

$$
H_{K}(\delta) \leq\left|D_{K}\right|^{1 / 2} H_{K}(\Lambda),
$$

where $\Lambda$ is a point in a projective space having as coordinates 1 and the coefficients of $f(X, Y)$ and $F(X, Y)$ (in any order). Put $\Delta=\max \{\operatorname{deg} f, \operatorname{deg} F\}$. By Lemma 2, there are polynomials $A(X, Y), B(X, Y) \in O_{K}[X, Y]$ and a non-zero algebraic integer $c$ of $K$, satisfying

$$
N_{K}(c)<\left(49 \Delta^{4}\right) ! H_{K}(\Lambda)^{49 \Delta^{4}},
$$

such that

$$
A(X, Y) f_{1}(X, Y)+B(X, Y) F_{1}(X, Y)=c .
$$

Let $x, y \in O_{K}$ such that $F(X, Y)=0$. It follows that $A(x, y) f_{1}(x, y)=c$. Since $A(x, y)$ and $f_{1}(x, y)$ are algebraic integers of $K$, we deduce that the integer $N_{K}\left(f_{1}(x, y)\right)$ divides $N_{K}(c)$. Thus we obtain

$$
N_{K}\left(f_{1}(x, y)\right) \leq\left(49 \Delta^{4}\right) ! H_{K}(\Lambda)^{49 \Delta^{4}} .
$$

Put $g_{1}(X, Y)=\delta-f_{1}(X, Y)$. Similarly we obtain

$$
N_{K}\left(g_{\mathrm{i}}(x, y)\right) \leq\left(49 \Delta^{4}\right) ! H_{K}\left(\Lambda^{*}\right)^{49 \Delta^{4}},
$$


where $\Lambda^{*}$ is a point in a projective space having as coordinates the coefficients of the polynomials $g_{1}(X, Y) / \delta=1-f(X, Y)$ and $F(X, Y)$. We have $H\left(\Lambda^{*}\right) \leq 2 H(\Lambda)$ Hence the algebraic integers $f_{1}(x, y)$ and $g_{1}(x, y)$ satisfy

$$
\frac{1}{\delta} f_{1}(x, y)+\frac{1}{\delta} g_{1}(x, y)=1
$$

and

$$
N_{K}\left(f_{1}(x, y)\right), N_{K}\left(g_{1}(x, y)\right)<\left(50 \Delta^{4} H(\Lambda)\right)^{49 d \Delta^{4}} .
$$

Put $A=\max \left\{e,\left|D_{K}\right|^{1 / 2} H_{K}(\Lambda)\right\}$ and $M=\left(50 \Delta^{4} H(\Lambda)\right)^{49 d \Delta^{4}}$. By Lemma 3 ,

$$
\max \left\{H_{K}\left(f_{1}(x, y)\right), H_{K}\left(g_{1}(x, y)\right)\right\}<\exp \left\{\Psi(d)\left|D_{K}\right|\left(\log *\left|D_{K}\right|\right)^{2 d-1} \log (M A)\right\} .
$$

Then

$$
\max \left\{H_{K}\left(f_{1}(x, y)\right), H_{K}\left(g_{1}(x, y)\right)\right\}<\exp \left\{C(d, \Delta)\left|D_{K}\right|\left(\log *\left|D_{K}\right|\right)^{2 d} \log * H_{K}(\Lambda)\right\},
$$

where

$$
C(d, \Delta)<1100 d^{11 d+13} 3^{4 d+29} \Delta^{5} .
$$

So $(x, y)$ is a solution to the equation

$$
G(X, Y)=f_{1}(X, Y)-\Gamma=0,
$$

where $\Gamma$ is an algebraic integer of $K$, having

$$
H_{K}(\Gamma)<\exp \left\{C(d, \Delta)\left|D_{K}\right|\left(\log *\left|D_{K}\right|\right)^{2 d} \log * H_{K}(\Lambda)\right\} .
$$

The height of $G(X, Y)$ satisfies $H(G)<2 H(\Lambda) H(\delta) H(\Gamma)$.

Let $R(X)$ be the resultant of the polynomials $F(X, Y)$ and $G(X, Y)$, considered as polynomials with coefficients in $K[X]$. If $R(X) \equiv 0$, then there exist two polynomials $S(X, Y)$ and $T(X, Y)$ with $\operatorname{deg}_{Y} S<\operatorname{deg}_{Y} G$ and $\operatorname{deg}_{Y} T<\operatorname{deg}_{Y} F$ such that

$$
S(X, Y) F(X, Y)=T(X, Y) G(X, Y) .
$$

The polynomial $F(X, Y)$ is irreducible and $\operatorname{deg}_{Y} T<\operatorname{deg}_{Y} F$. Then $F(X, Y)$ divides $G(X, Y)$. It follows that the function $\varphi$ is a constant, which is not true. Hence $R(X) \not \equiv 0$. Since $F(x, y)=0$ and $G(x, y)=0$, we have $R(x)=0$. By Lemmas 4 and 5 , we get

$$
H(x) \leq 2 H(R) \leq(2 \Delta(\Delta+1))^{2 \Delta}(H(F) H(G))^{\Delta} .
$$


Then

$$
H(x) \leq(2 \Delta(\Delta+1))^{2 \Delta}\left(2 H(\Lambda)^{2} H(\Gamma) H(\delta)\right)^{\Delta} .
$$

The same argument with the roles of $x$ and $y$ interchanged shows that the above bound holds for $H(y)$. Therefore we obtain

$$
\max \left\{H_{K}(x), H_{K}(y)\right\}<\exp \left\{\Omega_{1}(d, \Delta)\left|D_{K}\right|\left(\log *\left|D_{K}\right|\right)^{2 d} \log * H_{K}(\Lambda)\right\},
$$

where $\Omega_{1}(d, \Delta)<\Delta^{6} d^{11 d+13} 81^{d+9}$.

\section{Curves of genus 0}

Let $F(X, Y)$ be an absolutely irreducible polynomial in $K[X, Y]$ of degree $N$. Assume that the affine plane curve $C$ defined by the equation $F(X, Y)=0$ is nonsingular. Let $\bar{K}(C)$ be the function field of $C$ and $\Sigma_{\infty}$ the set of discrete valuation rings $U$ of $\bar{K}(C)$ such that $\bar{K} \subset U$ and $U \cap \bar{K}(X)$ is the discrete valuation ring of $\bar{K}(X)$ defined by $1 / X$. In this section we prove that if $C$ has genus 0 and $\Sigma_{\infty}$ contains at least three distinct elements, then the ring of regular functions of $C$ has an exceptional unit. Thus Theorem 1 implies an explicit upper bound for their integer points, improving on previous estimates given in [14] and in [1, Theorem 5B].

THEOREM 2. Let $F(X, Y)$ and $C$ be as above. Suppose that $C$ has genus 0 and the set $\Sigma_{\infty}$ contains at least three (distinct) elements. Then every solution $x, y \in O_{K}$ of the equation $F(X, Y)=0$ satisfies

$$
\max \left\{H_{K}(x), H_{K}(y)\right\}<\exp \left\{\Omega_{2}(d, N)\left|D_{K}\right|^{4 N^{3}} H_{K}(F)^{146 N^{13}}\right\},
$$

where $\Omega_{2}(d, N)<(2 N)^{2600 d N^{23}} d^{13 d N^{3}+14}$.

Let $\Sigma$ be the set of discrete valuation rings $U$ of $\bar{K}(C)$ such that $\bar{K} \subset U$. A divisor D on the curve $C$ is a formal sum

$$
\mathbf{D}=a_{1} V_{1}+\cdots+a_{s} V_{s}
$$

where $a_{1}, \ldots, a_{s}$ are rational integers and $V_{1}, \ldots, V_{s}$ elements of $\Sigma$. Given $f \in \bar{K}(C)$ and $V \in \Sigma$, let $\operatorname{ord}_{V}(f)$ denote the order of the function $f$ at $V$. Let $\mathbf{L}(\mathbf{D})$ be the set of functions $f \in \bar{K}(C)$ having $\operatorname{ord}_{V_{i}}(f) \geq-a_{i}$ and $\operatorname{ord}_{V}(f) \geq 0$ for every $V \in \Sigma$, with $V \neq V_{i}(i=1, \ldots, s)$. Then $\mathbf{L}(\mathbf{D})$ is a finite-dimensional vector space over $\bar{K}$ (see [8]). 
LEMMA 6. Assume that the curve $C$ has genus 0 and $N \geq 2$. Let $V \in \Sigma_{\infty}$. Then there are polynomials $g(X, Y)$ and $q(X)$ satisfying

$$
\begin{gathered}
\operatorname{deg} q<N^{2}, \quad \operatorname{deg}_{X} g \leq 4 N^{2}, \quad \operatorname{deg}_{Y} g<N, \\
H(q)<\left(6 N^{3} H(F)\right)^{2 N^{3}}, \quad H(Z)<\left(9 N^{4} H(F)\right)^{366 N^{\prime \prime},},
\end{gathered}
$$

where $Z$ is a point in a projective space having as coordinates 1 and the coefficients of $g(X, Y)$, such that the fraction $g(X, Y) / q(X)$ represent a function $\phi$ in $\mathbf{L}(\mathbf{V})-\bar{K}$. The leading coefficient of $q(X)$ is 1 and the coefficients of $g(X, Y)$ and $q(X)$ lie in an algebraic number field $L$, having $L \supseteq K,[L: K] \leq N$ and discriminant $D_{L}$ satisfying

$$
\left|D_{L}\right|<(2 N)^{865 d N^{21}}\left|D_{K}\right|^{N} H_{K}(F)^{48 N^{11}} .
$$

Let $U \in \Sigma_{\infty}-V$. Then there is $u \in \bar{K}$ with $H(u)<\left(9 N^{4} H(F)\right)^{376 N^{11}}$, such that the function $\phi-u$ has a zero at $U$. Furthermore, there is an algebraic number field $M$, with $M \supseteq K,[M: K] \leq N$ and discriminant $D_{M}$ satisfying

$$
\left|D_{M}\right|<(2 N)^{865 d N^{21}}\left|D_{K}\right|^{N} H_{K}(F)^{48 N^{13}},
$$

such that $u$ lies in the composite $L M$ of the fields $L$ and $M$.

PROOF. By the Riemann-Roch Theorem, the space $\mathbf{L}(\mathbf{V})$ has dimension 2. Theorem A2 of [16] implies that there are polynomials $q(X), g_{i}(X, Y)(i=1,2)$, such that the fractions $g_{i}(X, Y) / q(X)(i=1,2)$ represent a basis of the vector space $\mathbf{L}(\mathbf{V})$. By [15, Lemma 1.1], the divisor $V$ is defined over a field $L$ having $L \supseteq K$, and $[L: K] \leq N$. Furthermore, the discriminant $D_{L}$ of $L$ satisfies

$$
\left|D_{L}\right|<(2 N)^{865 d N^{21}}\left|D_{K}\right|^{N} H_{K}(F)^{48 N^{11}} .
$$

Then [16, Theorem B2] implies that the polynomials $g_{i}(X, Y)(i=1,2)$ and $q(X)$ have their coefficients in $L$.

Let $F_{Y}(X, Y)$ be the partial derivative of $F(X, Y)$ with respect $Y$. We denote by $R(X)$ the resultant of $F(X, Y)$ and $F_{Y}(X, Y)$ with respect to $Y$. By Lemma 6 , $H(R)<\left(3 N^{3} H(F)\right)^{2 N-1}$. Let $D(X)$ be the discriminant of $F(X, Y)$ considered as polynomial with coefficients in $K[X]$. By [16, Theorem A2], $\operatorname{deg} q \leq N(N-1)$ and the roots of $q(X)$ are among the roots of $D(X)$. Furthermore, we can assume that the leading coefficient of $q(X)$ is 1 . Let $q(X)=\left(X-a_{1}\right) \cdots\left(X-a_{r}\right)$. Since $D(X)$ divides $R(X)$, we have $R\left(a_{i}\right)=0(i=1, \ldots, r)$. By Lemma 4 ,

$$
H\left(a_{i}\right) \leq 2 H(R) \quad(i=1, \ldots, r) .
$$


Then [18, Theorem 5.9, p. 211], gives

$$
H(q) \leq 2^{N(N-1)} H\left(a_{1}\right) \cdots H\left(a_{r}\right) .
$$

Combining the above inequalities we deduce

$$
H(q) \leq(4 H(R))^{N(N-1)} \leq 4^{N(N-1)}\left(3 N^{3} H(F)\right)^{N(N-1)(2 N-1)}<\left(6 N^{3} H(F)\right)^{2 N^{3}} .
$$

By [16, Theorem A2], $\operatorname{deg}_{X} g_{i}<4 N^{2}$. Let $F(X, Y)=a_{0}(X) Y^{n}+a_{1}(X) Y^{n-1}+$ $\cdots+a_{n}(X)$. Following the notation of [16], we have

$$
g_{i}(X, Y)=\sum_{j=1}^{n} b_{i j}(X) y_{j}(X, Y) \quad(i=1,2),
$$

where $y_{1}(X, Y)=1$ and

$$
y_{j}(X, Y)=a_{0}(X) Y^{j-1}+a_{1}(X) Y^{j-2}+\cdots+a_{j-2}(X) Y \quad(j=2, \ldots, n)
$$

and $b_{i j}(X) \in L[X]$. From [16, pp. 204, 209 and 196], we get

$$
b_{i j}(X)=\delta_{i j 0}+\delta_{i j 1} X+\cdots+\delta_{i j,} X^{v}
$$

with $v<3 N^{2}$. By [16, Lemma 26], the vector $\delta_{i}=\left\{\delta_{i j p}\right\}_{1 \leq j \leq n, 0 \leq p \leq \nu}$ has absolute height $H\left(\delta_{i}\right)<\left(9 N^{4} H(F)\right)^{365 N^{\prime \prime}}$. We have

$$
\begin{aligned}
g_{i}(X, Y) & =\sum_{j=1}^{n} b_{i j}(X) y_{j}(X, Y) \\
& =b_{i 1}(X)+\left(b_{i 2} a_{0}(X)+\cdots+b_{i n}(X) a_{n-2}(X)\right) Y+\cdots+b_{i n}(X) a_{0}(X) Y^{n-1} .
\end{aligned}
$$

By the proof of [16, Theorem C2], we can choose a vector $\delta_{i}$ such that one of the $\delta_{i j p}$ is 1 . Let $Z_{i}$ be a point in a projective space having as coordinates 1 and the coefficients of $g_{i}(X, Y)$. We can suppose without loss of generality that one of the coefficients of $F(X, Y)$ is 1 . Then we obtain

$$
H\left(Z_{i}\right)<6 N^{4} H\left(\delta_{i}\right) H(F)<\left(9 N^{4} H(F)\right)^{366 N^{11}} .
$$

Since the dimension of the space $\mathrm{L}(\mathrm{V})$ is 2 , one of the fractions $g_{i}(X, Y) / q(X)(i=$ $1,2)$ represents a non-constant function on $C$. Denote this function by $\phi$.

Let $y$ be an algebraic function (in an algebraic closure of $\bar{K}(X)$ ) such that $F(X, y)=$ 0 . By the proof of [15, Lemma 1.1], the coefficients of a Puiseux expansion of the function $y$ at $U$ generate over $K$ an algebraic number field $M$ with degree $[M: K] \leq$ $N$. Furthermore, the discriminant $D_{M}$ of $M$ has

$$
\left|D_{M}\right|<(2 N)^{865 d N^{21}}\left|D_{K}\right|^{N} H_{K}(F)^{48 N^{11}} .
$$


Let $e$ be the ramification index at $U$. Put $X_{U}=1 / X^{1 / e}$. The function $\phi$ has a unique pole at $V$. Then $\operatorname{ord}_{U}(\phi) \geq 0$. So the Puiseux expansion of $\phi$ at $U$ is given by a series of the form

$$
\phi=u_{0}+u_{1} X_{U}+\cdots .
$$

It follows that the function $\phi-u_{0}$ has a zero at $U$. By [16, Theorem C2] (or more precisely by the proof of this theorem), the coefficients $u_{0}, u_{1}, \ldots$ are elements in $L M$. Furthermore, the same theorem yields $H\left(u_{0}\right)<\left(9 N^{4} H(F)\right)^{376 N^{11}}$.

LEMMA 7. Let $A(X, Y), B(X, Y) \in K[X, Y]$ be such that the fraction $A(X, Y) /$ $B(X, Y)$ defines a regular function $\phi$ on $C$. Put

$$
\rho=\operatorname{deg} A+\max \{\operatorname{deg} F, \operatorname{deg} B\}+16 \max \{\operatorname{deg} B, \operatorname{deg} F\}^{4} .
$$

Then the function $\phi$ is represented by a polynomial $P(X, Y) \in K[X, Y]$ on $C$, satisfying

$$
\operatorname{deg} P<\rho-\max \{\operatorname{deg} F, \operatorname{deg} B\} \quad \text { and } \quad H(\Pi) \leq\left(\rho^{2}\right) ! H(\Psi)^{\rho^{2}},
$$

where $\Psi$ is a point in a projective space having as coordinates the coefficients of $A(X, Y), B(X, Y), F(X, Y)$ and $\Pi$ is a point in a projective space having as coordinates 1 and the coefficients of $P(X, Y)$.

PROOF. Since the fraction $A(X, Y) / B(X, Y)$ defines a regular function on $C$, there are polynomials $P(X, Y)$ and $R(X, Y)$ in $K[X, Y]$ such that

$$
A(X, Y)=P(X, Y) B(X, Y)+R(X, Y) F(X, Y) .
$$

By [12, Appendix], we can take

$$
\operatorname{deg} P, \operatorname{deg} R \leq \operatorname{deg} A+16 \max \{\operatorname{deg} B, \operatorname{deg} F\}^{4}
$$

(the result of [12, Appendix] is given for the case $K=\mathbb{Q}$, but as one can easily see its proof remain the same when $K$ is an arbitrary algebraic number field). Write

$$
\begin{array}{ll}
A(X, Y)=\sum_{i=0, j=0}^{a, a^{\prime}} A_{i j} X^{i} Y^{j}, & B(X, Y)=\sum_{i=0, j=0}^{b, b^{\prime}} B_{i j} X^{i} Y^{j}, \\
P(X, Y)=\sum_{i=0, j=0}^{p, p^{\prime}} P_{i j} X^{i} Y^{j}, & R(X, Y)=\sum_{i=0, j=0}^{r, r^{\prime}} R_{i j} X^{i} Y^{j}
\end{array}
$$


and $F(X, Y)=\sum_{i=0 . j=0}^{m . n} F_{i j} X^{i} Y^{j}$. Then

$$
\sum_{s=0, \mathrm{~s}=0}^{S . T}\left(\sum_{i+k=s, j+l=t}\left(P_{i j} B_{k l}+R_{i j} F_{k l}\right)\right) X^{s} Y^{t}=\sum_{s=0, \mathrm{r}=0}^{a . a^{\prime}} A_{s l} X^{s} Y^{t}
$$

where

$$
S=\max \{p+b, r+m, a\} \quad \text { and } \quad T=\max \left\{p^{\prime}+b^{\prime}, r^{\prime}+n, a^{\prime}\right\} \text {. }
$$

Put $\pi=\operatorname{deg} A+16 \max \{\operatorname{deg} B, \operatorname{deg} F\}^{4}$. We have

$$
S, T \leq \pi+\max \{\operatorname{deg} F, \operatorname{deg} B\} .
$$

Consider the homogeneous linear system

$$
\sum_{i+k=s, j+l=t}\left(X_{i j} B_{k l}+Y_{i j} F_{k l}\right)-Z A_{s t}=0 \quad(s=0, \ldots, S, t=0, \ldots, T)
$$

in unknowns $X_{i j}\left(i=0, \ldots, p, j=0, \ldots, p^{\prime}\right), Y_{i j}\left(i=0, \ldots, r, j=0, \ldots, r^{\prime}\right)$ and $Z$. There are at most $1+S T$ equations. The numbers $P_{i j}(i=0, \ldots, p, j=$ $\left.0, \ldots, p^{\prime}\right), R_{i j}\left(i=0, \ldots, r, j=0, \ldots, r^{\prime}\right)$ and 1 are a solution of the above system in $K$. By the proof of [11, Lemma 4, p. 442] there is a non-trivial solution of the system in $K, \pi_{i j}\left(i=0, \ldots, p, j=0, \ldots, p^{\prime}\right), \theta_{k l}\left(k=0, \ldots, r, l=0, \ldots, r^{\prime}\right)$ and $\zeta \neq 0$, such that

$$
\left|\pi_{i j}\right|_{v},\left|\theta_{k l}\right|_{v},|\zeta|_{v}<\max \left\{|A|_{v},|B|_{v},|F|_{v}\right\}^{S T} v((S T) !)
$$

for every $v \in M(K)$. The quantity $v((S T)$ !) is equal to $(S T)$ ! if $v$ corresponds to an archimedean absolute value and 1 otherwise.

The polynomials

$$
\Xi(X, Y)=\sum_{i=0, j=0}^{p, p^{\prime}}\left(\pi_{i j} / \zeta\right) X^{i} Y^{j} \quad \text { and } \quad \Theta(X, Y)=\sum_{i=0, j=0}^{r . r^{\prime}}\left(\theta_{i j} / \zeta\right) X^{i} Y^{j}
$$

satisfy

$$
A(X, Y)=\Xi(X, Y) B(X, Y)+\Theta(X, Y) F(X, Y)
$$

Furthermore, denote by $\Psi$ a point in a projective space having as coordinates the coefficients of $A(X, Y), B(X, Y), F(X, Y)$ and $\Phi$ a point in a projective space having as coordinates $1, \pi_{i j} / \zeta\left(i=0, \ldots, p, j=0, \ldots, p^{\prime}\right)$ and $\theta_{i j} / \zeta(i=0, \ldots, r, j=$ $\left.0, \ldots, r^{\prime}\right)$. Then we deduce

$$
H(\Phi) \leq H(\Psi)^{S T}(S T) !
$$


PROOF OF THEOREM 2. According to our assumptions, the set $\Sigma_{\infty}$ contains three distinct elements, say $V_{1}, V_{2}$ and $V_{3}$. By Lemma 6 , there are polynomials $g(X, Y)$ and $q(X)$ satisfying

$$
\operatorname{deg} q<N^{2}, \quad \operatorname{deg}_{X} g \leq 4 N^{2}, \quad \operatorname{deg}_{Y} g<N
$$

and

$$
H(q)<\left(6 N^{3} H(F)\right)^{2 N^{3}}, \quad H(Z)<\left(9 N^{4} H(F)\right)^{366 N^{\prime \prime}},
$$

where $Z$ is a point in a projective space having as coordinates 1 and the coefficients of $g(X, Y)$, such that the fraction $g(X, Y) / q(X)$ represents a function $\phi$ of the space $\mathbf{L}\left(V_{1}\right)-K$. The leading coefficient of the polynomial $q(X)$ is 1 . Furthermore, there are $u_{2}, u_{3} \in \bar{K}$ with

$$
H\left(u_{i}\right)<\left(9 N^{4} H(F)\right)^{376 N^{11}},
$$

such that the function $\phi-u_{i}$ has a zero at $V_{i}(i=2,3)$. The elements $u_{2}, u_{3}$ and the coefficients of $g(X, Y)$ and $q(X)$ lie in an algebraic number field $M$, having $M \supseteq K$ and $[M: K] \leq N^{3}$. The discriminant $D_{M}$ of $M$ has

$$
\left|D_{M}\right|<\left((2 N)^{865 d N^{21}}\left|D_{K}\right|^{N} H_{K}(F)^{48 N^{11}}\right)^{3 N^{2}} .
$$

Suppose $u_{2}=u_{3}$. Then the function $\phi-u_{2}$ has zeros at $V_{2}$ and $V_{3}$ and a unique pole at $V_{1}$ of order 1 , a contradiction. Hence $u_{2} \neq u_{3}$.

The function $\psi=\left(\phi-u_{2}\right) /\left(u_{3}-u_{2}\right)$ has a unique pole at $V_{1}$ of order 1 and a zero at $V_{2}$. It follows that $\psi$ has no other pole at any $U \in \Sigma-\Sigma_{\infty}$. Hence $\psi$ is a unit in $U$, for every $U \in \Sigma-\Sigma_{\infty}$. Since the affine curve $C$ is non-singular, the discrete valuation rings of $\Sigma-\Sigma_{\infty}$ are exactly the rings of regular functions $V_{P}$ at the points $P$ of the affine curve $C$. Then $\psi$ is a unit in $V_{P}$, for every $P \in C$. It follows that $\psi$ is a unit in the ring $\bigcap_{P \in C} V_{P}=\bar{K}[C]$. Furthermore, the function $1-\psi$ has a unique pole at $V_{1}$ of order 1 and a zero at $V_{3}$. Similarly we obtain that $1-\psi$ is a unit in $\bar{K}[C]$. Hence $\psi$ is an exceptional unit and therefore we can apply Theorem 1. By Lemma 7, there is a polynomial $P(X, Y)$ with coefficients in $M$, such that the function $\psi$ is represented by $P(X, Y)$ on $C$. Furthermore, $\operatorname{deg} P<17 N^{8}$ and $H(\Pi)<\left(289 N^{16} H(\Theta)\right)^{289 N^{16}}$, where $\Pi$ is a point in a projective space having as coordinates 1 and the coefficients of $P(X, Y)$ and $\Theta$ a point in a projective space having as coordinates the coefficients of $g(X, Y)-u_{2} q(X),\left(u_{2}-u_{3}\right) q(X)$ and $F(X, Y)$. We may assume, without loss of generality, that one of the coefficients of $F(X, Y)$ is 1 . Since the leading coefficient of $q(X)$ is 1 , we get

$$
H(\Theta)<4 H(Z) H(q)^{2} H(F) H\left(u_{2}\right)^{2} H\left(u_{3}\right)<\left(9 N^{4} H(F)\right)^{1495 N^{\prime \prime}} .
$$


Therefore $H(\Pi)<\left(9 N^{4} H(F)\right)^{432056 N^{27}}$. Let $x, y \in O_{K}$ such that $F(x, y)=0$. Then Theorem 1 gives

$$
\max \left\{H_{K}(x), H_{K}(y)\right\}<\exp \left\{C(d, N)\left|D_{K}\right|^{4 N^{3}} H_{K}(F)^{146 N^{13}}\right\},
$$

where $C(d, N)<(2 N)^{2600 d N^{23}} d^{13 d N^{3}+14}$. (To conclude this bound we have used the inequality $\log * n \leq m n^{1 / m}$ for every positive integers $n$ and $m$.)

\section{Elliptic equations}

In this section we obtain an explicit upper bound for the height of integral solutions to the elliptic equations, reducing this problem to the same problem for curves having an exceptional unit. Then Theorem 1 implies a bound for the height of integral solutions to the elliptic equations, improving on the estimate obtained recently by Schmidt in [17].

THEOREM 3. Suppose $f(X)=X^{3}+a X+b$ has coefficients in $O_{K}$ and discriminant $\Delta(f)=-\left(4 a^{3}+27 b^{2}\right) \neq 0$. Then all solutions $(x, y) \in O_{K}^{2}$ to the equation $Y^{2}=f(X)$ satisfy

$$
\max \left\{H_{K}(x), H_{K}(y)\right\}<\exp \left\{\Omega_{3}(d)\left|D_{K}\right|^{25}\left|N_{K}(\Delta(f))\right|^{27} \log * H_{K}(f)\right\},
$$

where $\Omega_{3}(d)<10^{585 d+46} d^{312 d+13}$.

COROllary 1. Suppose $f(X)=X^{3}+a X^{2}+b x+c$ has coefficients in $O_{K}$ and discriminant $\Delta(f)=-\left(4 a^{3} c+4 b^{3}+27 c^{2}-18 a b c-a^{2} b^{2}\right) \neq 0$. Then all solutions $(x, y) \in O_{K}^{2}$ to the equation $Y^{2}=f(X)$ satisfy

$$
\max \left\{H_{K}(x), H_{K}(y)\right\}<\exp \left\{\Omega_{4}(d)\left|D_{K}\right|^{25}\left|N_{K}(\Delta(f))\right|^{27} \log * H_{K}(f)\right\},
$$

where $\Omega_{4}(d)<10^{740 d+48} d^{312 d+13}$.

PROOF OF THEOREM 3. We shall use an argument that goes back to an idea of Chabauty (see [7, p. 140]). Consider the elliptic curve

$$
E: Y^{2}=X^{3}+a X+b .
$$

Let $(x, y)$ be a point on $E$ with coordinates in $\bar{K}$, such that $2(x, y) \neq 0$. Then $2(x, y)=(\phi(x, y), \psi(x, y))$, where $\phi(x, y)=-2 x+\left(\frac{3 x^{2}+a}{2 y}\right)^{2} \quad$ and $\quad \psi(x, y)=-y+\left(\frac{3 x^{2}+a}{2 y}\right)(x-\phi(x, y))$. 
Let $S$ and $T$ be algebraic functions, in an algebraic closure of the function field $\bar{K}(E)$ of $E$, such that $X=\phi(S, T)$ and $Y=\psi(S, T)$. Setting $U=\left(3 S^{2}+a\right) /(2 T)$, we get

$$
X=-2 S+U^{2} \text { and } Y=-\frac{3 S^{2}+a}{2 U}+U\left(3 S-U^{2}\right) .
$$

Eliminating $S$ we deduce the equation

$$
\Phi(X, Y, U)=U^{4}-6 X U^{2}-8 Y U-3 X^{2}-4 a=0 .
$$

Let $(x, y) \in O_{K}^{2}$ be an integer point on the elliptic curve $E$, with $y \neq 0$. Let $u$ be a non-zero root of the equation $\Phi(x, y, U)=0$. Then $u$ is an algebraic integer. Put $s=\left(u^{2}-x\right) / 2$. Substituting $-2 s+u^{2}$ for $x$ in $\Phi(x, y, u)=0$, we get

$$
x=-2 s+u^{2} \quad \text { and } \quad y=-\frac{3 s^{2}+a}{2 u}+u\left(3 s-u^{2}\right) .
$$

Substituting this in $y^{2}=x^{3}+a x+b$, we deduce

$$
G(s, u)=9 s^{4}-4 u^{2} s^{3}+6 a s^{2}-4 a s u^{2}-4 b u^{2}+a^{2}=0 .
$$

Replacing $u^{2}$ by $2 s+x$ we obtain

$$
\Theta(x, s)=s^{4}-4 x s^{3}-2 a s^{2}-4 a x s-8 b s-4 b x+a^{2}=0 .
$$

It follows that $s$ is an algebraic integer.

Let $e_{1}, e_{2}, e_{3}$ be the roots of the equation $S^{3}+a S+b=0$. Consider the polynomial

$$
G(S, U)=-4 U^{2}\left(S^{3}+a S+b\right)+9 S^{4}+6 a S^{2}+a^{2} .
$$

Since $\left(9 S^{4}+6 a S^{2}+a^{2}\right) /\left(S^{3}+a S+b\right)$ is not a square in $\bar{K}(S)$, it follows that the algebraic curve $G(S, U)=0$ is irreducible. Let $e_{i}$ be a root of the polynomial $9 S^{4}+6 a S^{2}+a^{2}=\left(3 S^{2}+a\right)^{2}$. Then $e_{i}$ is a double root of the polynomial $S^{3}+a S+b$ which is not true. Thus, $S^{3}+a S+b$ and $9 S^{4}+6 a S^{2}+a^{2}$ have no common root. If the regular function $S-e_{i}$ on the affine curve $G(S, U)=0$ had a finite zero, then the polynomials $S^{3}+a S+b$ and $9 S^{4}+6 a S^{2}+a^{2}$ would have a common root, which is a contradiction. Thus $1 /\left(S-e_{i}\right)$ is a regular function on $G(S, U)=0$. Therefore the functions $S-e_{i}(i=1,2,3)$ are units in the ring of regular functions of the curve $G(S, U)=0$. Since

$$
\left(S-e_{1}\right)+\left(e_{2}-S\right)=e_{2}-e_{1},
$$

we obtain that the function $\left(S-e_{1}\right) /\left(e_{2}-e_{1}\right)$ is an exceptional unit.

Let $L=K(u)$. The equality $x=-2 s+u^{2}$ implies $s \in L$. Since $u$ satisfies the equation $\Phi(x, y, U)=0$, we have $[L: K] \leq 4$. Let $P(U)$ be the irreducible 
polynomial of $u$ over $K$ and $\Delta(P)$ the discriminant of $P(U)$. Since $u$ is an integer in $L, \Delta(P)$ is an integer in $K$ and the discriminant ideal $D_{L / K}$ of $L$ over $K$ divides $\Delta(P)$. Further, $P(U)$ divides $\Phi(x, y, U)$. The discriminant of the polynomial $\Phi(x, y, U)$ is $2^{12} \Delta(f)$. It follows that $D_{L / K}$ divides $2^{12} \Delta(f)$ (in $O_{K}$ ). Hence

$$
\left|N_{K}\left(D_{L / K}\right)\right| \leq 2^{12 d}\left|N_{K}(\Delta(f))\right| .
$$

Then the discriminant $D_{L}$ of $L$ satisfies

$$
\left|D_{L}\right| \leq\left|D_{K}\right|^{4}\left|N_{K}\left(D_{L / K}\right)\right| \leq 2^{12 d}\left|D_{K}\right|^{4}\left|N_{K}(\Delta(f))\right| .
$$

Denote by $M$ the field generated over $L$ by $e_{1}, e_{2}$ and $e_{3}$. Let $Q(X)$ be the irreducible polynomial of $e_{1}$ over $L$ and $\Delta(Q)$ the discriminant of $Q(X)$. Then $\Delta(Q)$ divides $\Delta(f)$. Since $e_{1}$ is an algebraic integer, the discriminant ideal $D_{L\left(e_{1}\right) / L}$ of $L\left(e_{1}\right)$ over $L$ divides $\Delta(Q)$. Thus $D_{L\left(e_{1}\right) / L}$ divides $\Delta(f)$. Hence

$$
\left|D_{L\left(e_{1}\right)}\right| \leq\left|D_{L}\right|^{3}\left|N_{L}\left(D_{L\left(e_{1}\right) / L}\right)\right| \leq\left|D_{L}\right|^{3}\left|N_{L}(\Delta(f))\right|=\left|D_{L}\right|^{3}\left|N_{K}(\Delta(f))\right|^{4} .
$$

Similarly we get

$$
\left|D_{M}\right| \leq\left|D_{L\left(e_{1}\right)}\right|^{2}\left|N_{K}(\Delta(f))\right|^{12} .
$$

Combining the two inequalities we obtain

$$
\left|D_{M}\right| \leq\left|D_{L}\right|^{6}\left|N_{K}(\Delta(f))\right|^{20} \leq 2^{72 d}\left|D_{K}\right|^{24}\left|N_{K}(\Delta(f))\right|^{26} .
$$

Let $\Lambda$ be a point in a projective space having as coordinates $1,1 /\left(e_{2}-e_{1}\right)$, $e_{1} /\left(e_{2}-e_{1}\right)$ and the coefficients of the polynomials $G(S, U)$. We easily deduce $H(\Lambda)<144 H(f)^{5}$. Theorem 1 yields

$$
\max \left\{H_{M}(u), H_{M}(s)\right\}<\exp \left\{C(d)\left|D_{K}\right|^{25}\left|N_{K}(\Delta(f))\right|^{27} \log * H_{K}(f)\right\},
$$

where $C(d)<10^{585 d+44} d^{312 d+13}$.

The equation $\Theta(x, s)=0$ implies

$$
x=\frac{s^{4}-2 a s^{2}-8 b s+a^{2}}{4\left(s^{3}+a s+b\right)} .
$$

Then

$$
H_{M}(x) \leq 770^{24 d} H_{M}(s)^{11} H_{K}(f)^{144} .
$$

Therefore

$$
\max \left\{H_{K}(x), H_{K}(y)\right\}<\exp \left\{12 C(d)\left|D_{K}\right|^{25}\left|N_{K}(\Delta(f))\right|^{27} \log * H_{K}(f)\right\} .
$$


Proof OF COROLlaRY 1. Consider the equation $Y^{2}=f(X)$ and multiply the two members by $3^{6}$. Putting $T=27 Y$ and $S=9 X$, we get

$$
T^{2}=S^{3}+3^{2} a S^{2}+3^{4} b S+3^{6} c .
$$

Furthermore, setting $S=U-3 a$, we obtain the equation

$$
T^{2}=g(U),
$$

where

$$
g(U)=U^{3}+3^{3}\left(3 b-a^{2}\right) U+3^{3}\left(2 a^{3}-3^{2} a b+3^{3} c\right) .
$$

Let $(x, y) \in O_{K}{ }^{2}$ with $y^{2}=f(x)$. Then $u=3 a+9 x$ and $t=27 y$ lie in $O_{K}$ and satisfy $t^{2}=g(u)$. We have $H(g) \leq 1026 H(f)^{3}$ and the discriminant of $g(U)$ is

$$
\Delta(g)=-\left[4\left(3^{3}\left(3 b-a^{2}\right)\right)^{3}+27\left(3^{3}\left(2 a^{3}-3^{2} a b+3^{3} c\right)\right)^{2}\right]=3^{12} \Delta(f) .
$$

Then Theorem 1 yields

$$
\max \left\{H_{K}(u), H_{K}(t)\right\}<\exp \left\{\Omega_{3}(d) 3^{324 d} 10\left|D_{K}\right|^{25}\left|N_{K}(\Delta(f))\right|^{27} \log * H_{K}(f)\right\} .
$$

Since $x=(u-3 a) / 9$ and $y=t / 27$, we obtain

$$
\max \left\{H_{K}(x), H_{K}(y)\right\}<\exp \left\{\Omega_{3}(d) 3^{324 d} 20\left|D_{K}\right|^{25}\left|N_{K}(\Delta(f))\right|^{27} \log * H_{K}(f)\right\} .
$$

\section{Thue curves}

By a Thue curve we mean a plane curve $g(X, Y)=b$, where $g(X, Y)$ is a binary form in $K[X, Y]$ with at least three pairwise distinct linear factors and $b$ a non-zero element of $K$. In this section we consider a more general class than Thue curves and we prove that the curves from this class have exceptional units. Thus Theorem 1 gives an upper bound for their integer points. Our estimate is sharper than that of Theorem $5 \mathrm{E}$ of $[1]$.

THEOREM 4. Let $F(X, Y)$ be an absolutely irreducible polynomial in $K[X, Y]$ of degree $N$, of the form

$$
F(X, Y)=\left(X-a_{1} Y\right)\left(X-a_{2} Y\right)\left(X-a_{3} Y\right) f(X, Y)+b,
$$

where $f(X, Y) \in K[X, Y], a_{1}, a_{2}, a_{3}, b \in K$ and the elements $a_{1}, a_{2}, a_{3}$ are pairwise distinct. Then every solution $x, y \in O_{K}$ of the equation $F(X, Y)=0$ satisfies

$$
\begin{aligned}
\max & \left\{H_{K}(x), H_{K}(y)\right\} \\
& <\exp \left\{\Omega_{5}(d, N)\left|D_{K}\right|\left(\log *\left|D_{K}\right|\right)^{2 d} \log *\left(H_{K}\left(1, a_{1}, a_{2}, a_{3}\right) H_{K}(F)\right)\right\},
\end{aligned}
$$

where $\Omega_{5}(d, N)<10^{30} 81^{d} N^{33} d^{11 d+13}$. 
ProOF. Denote by $C$ the algebraic curve defined by the equation $F(X, Y)=0$. Since the polynomials $\left(X-a_{1} Y\right)\left(X-a_{2} Y\right)\left(X-a_{3} Y\right)$ and $F(X, Y)$ have no common root in $\bar{K}^{2}$, we deduce that the fractions

$$
\frac{a_{3}-a_{1}}{a_{2}-a_{1}} \frac{X-a_{2} Y}{X-a_{3} Y} \quad \text { and } \quad \frac{a_{2}-a_{3}}{a_{2}-a_{1}} \frac{X-a_{1} Y}{X-a_{3} Y}
$$

define two units in $K[C]$ that will be denoted respectively by $\phi$ and $\psi$. It is easily verified that $\phi+\psi=1$. Thus $\phi$ is an exceptional unit. We can suppose without loss of generality that one of the coefficients of $F(X, Y)$ is 1 . By Lemma 7, the function $\phi$ is represented by a polynomial $P(X, Y)$ of $K[X, Y]$ satisfying $\operatorname{deg} P<17 N^{4}$ and

$$
H(\Pi)<\left(289 N^{8}\right) !\left(2 H\left(1, a_{1}, a_{2}, a_{3}\right)^{2} H(F)\right)^{289 N^{8}},
$$

where $\Pi$ is a point in a projective space having as coordinates $I$ and the coefficients of $P(X, Y)$. Let $\Lambda$ be a point in a projective space having as coordinates 1 and the coefficients of $P(X, Y)$ and $F(X, Y)$ (in any order). Then

$$
H(\Lambda) \leq H(\Pi) H(F)<\left(290 N^{8} H\left(1, a_{1}, a_{2}, a_{3}\right)^{2} H(F)\right)^{290 N^{8}} .
$$

Now we can apply Theorem 1 . Thus, if $x, y \in O_{K}$ such that $F(X, Y)=0$, we have

$$
\begin{aligned}
\max & \left\{H_{K}(x), H_{K}(y)\right\} \\
& <\exp \left\{C(d, N)\left|D_{K}\right|\left(\log *\left|D_{K}\right|\right)^{2 d} \log *\left(H_{K}\left(1, a_{1}, a_{2}, a_{3}\right) H_{K}(F)\right)\right\},
\end{aligned}
$$

where $C(d, N)<10^{30} 81^{d} N^{33} d^{11 d+13}$.

\section{Acknowledgement}

The author wishes to thank the referee for several helpful suggestions and comments.

\section{References}

[1] Y. Bilu, Effective analysis of integral points on algebraic curves (Thesis, Beer Sheva, 1993).

[2] — 'Effective analysis of integral points on algebraic curves', Israel J. Math. (1995), 235-252.

[3] W. D. Brownawell, 'Bounds for the degree in the Nullstellensatz', Ann. of Math. 126 (1987), 577-591.

[4] Y. Bugeaud and K. Györy, 'Bounds for the solutions of unit equations', Acta Arith. 74 (1996), 67-80.

[5] D. Kubert and S. Lang, 'Units in the modular function fields I', Math. Ann. 218 (1975), 67-96. 
[6] - Modular units (Springer, Berlin, 1981).

[7] S. Lang, Elliptic curves. Diophantine analysis (Springer, Berlin, 1978).

[8] - Introduction to algebraic and abelian functions (Springer, Berlin, 1982).

[9] - Fundamentals of Diophantine geometry (Springer, Berlin, 1983).

[10] A. F. Lavrik, 'A remark on the Siegel-Brauer theorem concerning the parameters of algebraic number fields', Mat. Zametki 8 (1970), 259-263 (in Russian). (English Trans.: Math. Notes 8 (1970), 615-617).

[11] D. W. Masser and G. Wüstholz, 'Fields of large trancendence degree generated by values of elliptic functions', Invent. Math. 72 (1983), 407-464.

[12] E. W. Mayr and A. R. Meyer, 'The complexity of the word problems for commutative semigroups and polynomial ideals', Adv. Math. 46 (1982), 305-329.

[13] M. Mignotte, 'An inequality of the greatest roots of a polynomial', Elem. Math. 46 (1991), 85-86.

[14] D. Poulakis, 'Points entiers sur les courbes de genre 0', Colloq. Math. 62 (1992), 1-7.

[15] _ 'Points entiers et modèles des courbes algeébriques', Monatsh. Math. 118 (1994), 111-143.

[16] W. M. Schmidt, 'Construction and estimation of bases in function fields', J. Number Theory 39 (1991), 181-224.

[17] —, 'Integer points on curves of genus 1', Compositio Math. 81 (1992), 33-59.

[18] J. H. Silverman, The arithmetic of elliptic curves (Springer, Berlin, 1986).

[19] P. Voutier, 'An upper bound for the size of integral solutions to $Y^{m}=f(X)$ ', J. Number Theory 55 (1995), 247-271.

[20] - 'An effective lower bound for the height of algebraic numbers', Acta Arithm. 74 (1996), $81-95$.

Department of Mathematics

Aristotle University of Thessaloniki

54006 Thessaloniki

Greece 